\title{
Scalar-Gauss-Bonnet theories: evasion of no-hair theorems and novel black-hole solutions
}

\section{Panagiota Kanti*}

Division of Theoretical Physics, Department of Physics, University of Ioannina, Ioannina GR-45110, Greece

E-mail: pkantidcc.uoi.qn

\section{Athanasios Bakopoulos}

Division of Theoretical Physics, Department of Physics, University of Ioannina, Ioannina

GR-45110, Greece

E-mail: abakopdcc.uoi.gr

\section{Nikolaos Pappas}

Nuclear and Particle Physics Section, Physics Department, National and Kapodistrian

University of Athens, Athens GR-15771, Greece

E-mail: npappasecc.uoi.gn

\begin{abstract}
We consider a generalised gravitational theory that contains the Ricci scalar curvature and a scalar field coupled to the higher-derivative, quadratic Gauss-Bonnet gravitational term through an arbitrary coupling function $f(\phi)$. We review both of the existing no-hair theorems, the old and the novel, and show that these are easily evaded; this opens the way for black holes to emerge in the context of this theory. Indeed, we demonstrate that, under mild only assumptions for $f(\phi)$, we may construct asymptotic solutions that describe either a regular black-hole horizon or an asymptotically-flat solution. We then demonstrate, through numerical integration, that these asymptotic solutions may be smoothly connected and that novel, regular black-hole solutions with non-trivial scalar hair emerge for any form of the coupling function $f(\phi)$. We present and discuss the physical characteristics of a large number of such solutions for a plethora of coupling functions $f(\phi)$. Finally, we consider the pure scalar-Gauss-Bonnet theory, under the assumption that the Ricci scalar may be ignored, and we investigate whether novel black-hole solutions may arise in this case.
\end{abstract}

Corfu Summer Institute 2018 "School and Workshops on Elementary Particle Physics and Gravity" (CORFU2018)

31 August - 28 September, 2018

Corfu, Greece

\footnotetext{
* Speaker.
} 


\section{Introduction}

Einstein's theory of General Relativity is a mathematically beautiful and experimentally tested theory of gravity. But it is not a perfect theory - if such a theory really exists. As is well known, the Standard Cosmological Model has a number of open problems: the nature of dark matter and dark energy, the coincidence problem, the spacetime singularities, the right model for inflation, to name only a few. On the gravity side, General Relativity predicts the existence of only three families of black-hole solutions and admits no stable wormhole solutions. Also, the unification of gravity with the other forces in nature seems unlikely within its current mathematical framework - General Relativity is based on tensors and is not a renormalizable theory in contrast with the renormalizable gauge field theories that are formulated in the flat Minkowski spacetime in terms of four-vectors.

Perhaps, all these accumulated problems and open questions point to the need for changing the theoretical framework for gravity. During the last few decades, the so-called generalised gravitational theories, where additional fields and higher gravitational terms may be present, have attracted the interest of many scientists. These theories comprise a popular test-bed for the formulation of the ultimate theory of gravity beyond Einstein's General Theory of Relativity, and are thus under intense research activity. These theories are of course not new; generalised gravitational theories have emerged over the years from either the string effective action at low energies [W] or as part of a Lovelock effective theory []] in four dimensions or even in the form of the recently revived scalartensor Horndeski/Galileon [ [3] theories. But, then, how are the predictions of General Relativity affected by the presence of those additional contributions to the theory? Can we find many novel black-hole solutions beyond the limits of General Relativity? Are the known black-hole solutions of General Relativity not valid any more?

In the context of General Relativity, the black-hole solutions have been indeed uniquely determined and classified according to their properties (mass, charge and angular-momentum). No-Hair theorems, that forbid the association of a black hole with any other "charge" or field, were formulated quite early on. The existence of black-hole solutions associated with a non-trivial scalar field in the region outside the black-hole horizon has also been intensively studied. The old no-hair theorem [䧃] was formulated in the seventies, and excluded static black holes with a scalar field. However, this was outdated by the discovery of black holes with Yang-Mills [[]], Skyrme fields [圆] or conformally-coupled scalar fields [ $[\square]$. Twenty years later, the novel no-hair theorem [ $[$ ] ] was formulated (for more recent analyses, see [四, 四, 四]). But this was also shown to be evaded: the

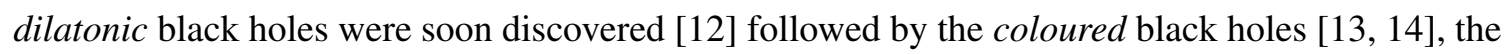
rotating black holes [피, [6] and more recently the black holes in the context of the shift-symmetric Galileon theory [1]7, [8]].

Compared, however, to the possibilities that the concept of a generalised gravitational theory opens up, the number of novel black-hole solutions that have been found is relatively small. The question then comes up: can we extend this 'elite' group of theories that evade the no-hair theorems and thus find new black-hole solutions? In this work, we will consider a class of gravitational theories where a scalar field $\phi$ has a general coupling function $f(\phi)$ to the quadratic gravitational Gauss-Bonnet (GB) term. It is, in fact, in the context of this theory that the dilatonic

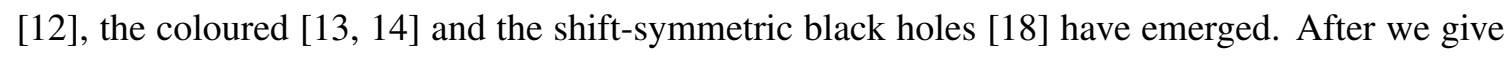
some further motivation for the chosen gravitational theory, we will investigate whether our theory 
may evade the existing no-hair theorems for an arbitrary form of the scalar-GB coupling function $f(\phi)$, and allow for the emergence of novel black-hole solutions, with a regular horizon and an asymptotically-flat limit. We will review some of the recently derived results in the literature [एव, [20, [2] ] and discuss the characteristics of these novel solutions. At the final part of this work, we will also investigate whether solutions may arise in the context of the pure scalar-Gauss-Bonnet theory where the Ricci scalar may be ignored.

\section{The Einstein-Scalar-Gauss-Bonnet theory}

In this work, we will therefore consider the following generalised gravitational theory

$$
S=\frac{1}{16 \pi} \int d^{4} x \sqrt{-g}\left[R-\frac{1}{2} \partial_{\mu} \phi \partial^{\mu} \phi+f(\phi) R_{G B}^{2}\right],
$$

where the GB term is defined as

$$
R_{G B}^{2}=R_{\mu v \rho \sigma} R^{\mu v \rho \sigma}-4 R_{\mu v} R^{\mu v}+R^{2}
$$

in terms of the Ricci scalar curvature $R$, the Ricci tensor $R_{\mu \nu}$ and the Riemann tensor $R_{\mu v \rho \sigma}$.

This theory has a number of attractive points. First, apart from the linear Ricci term, it contains a quadratic gravitational term i.e. the next important term in a strong-curvature regime such as near a black-hole horizon; nevertheless, it leads to field equations with only up to 2nd-order derivatives, and with no Ostrogradski instabilities [22]. In addition, it is a very "rich" theory, since:

- if we set $f(\phi) \sim \ln \left[2 e^{\phi} \eta^{4}\left(i e^{\phi}\right)\right]$, where $\eta(x)$ is the so-called Dedekind function, we recover the moduli part of the heterotic superstring effective action at low energies. It was in the context of this theory that singularity-free cosmological solutions emerged [22]];

- if we set $f(\phi) \sim e^{\phi}$, we recover the dilatonic superstring effective theory in the context of which the dilatonic black holes [ㅁ] ] and shortly afterwards the coloured black holes [피, [4]] (with the addition of a Yang-Mills field) were discovered. Also, in the context of the same theory, stable, traversable wormhole solutions were found in [24];

- if we set $f(\phi) \sim \phi$, then we recover the shift-symmetric Galileon theory, in the context of which the corresponding static black holes arise [एष]].

Choosing therefore the form of the coupling function between the scalar field and the GB term to have a specific form, particular forms of the theory (‥J) are obtained each one leading to interesting families of either gravitational or cosmological solutions. Here, we will focus on the case of black holes and pose the following question: for what other forms, apart from the exponential and linear one, one may find novel black-hole solutions? In order to find the answer to this question, we will keep the form of $f(\phi)$ arbitrary, and we will look for regular, static, spherically-symmetric and asymptotically-flat black-hole solutions. These will be described by the line-element

$$
d s^{2}=-e^{A(r)} d t^{2}+e^{B(r)} d r^{2}+r^{2}\left(d \theta^{2}+\sin ^{2} \theta d \varphi^{2}\right)
$$


where $A(r)$ and $B(r)$ are unknown functions of only the radial coordinate. The scalar field $\phi$ will also be assumed to be static and spherically-symmetric and thus to depend only on $r$, too.

By varying the above action with respect to the metric tensor and scalar field, we obtain the following gravitational field equations and the equation for the scalar field:

$$
G_{\mu \nu}=T_{\mu \nu}, \quad \nabla^{2} \phi+\dot{f}(\phi) R_{G B}^{2}=0,
$$

respectively, where a dot over the coupling function $f$ denotes the derivative with respect to the scalar field. The energy-momentum tensor has the form

$$
T_{\mu v}=-\frac{1}{4} g_{\mu v} \partial_{\rho} \phi \partial^{\rho} \phi+\frac{1}{2} \partial_{\mu} \phi \partial_{\nu} \phi-\frac{1}{2}\left(g_{\rho \mu} g_{\lambda v}+g_{\lambda \mu} g_{\rho v}\right) \eta^{\kappa \lambda \alpha \beta} \tilde{R}_{\alpha \beta}^{\rho \gamma} \nabla_{\gamma} \partial_{\kappa} f,
$$

where

$$
\tilde{R}_{\alpha \beta}^{\rho \gamma}=\eta^{\rho \gamma \sigma \tau} R_{\sigma \tau \alpha \beta}=\varepsilon^{\rho \gamma \sigma \tau} R_{\sigma \tau \alpha \beta} / \sqrt{-g} .
$$

Using the form of the line-element (Dי]), the Einstein's equations take the following explicit form

$$
\begin{gathered}
4 e^{B}\left(e^{B}+r B^{\prime}-1\right)=\phi^{2}\left[r^{2} e^{B}+16 \ddot{f}\left(e^{B}-1\right)\right]-8 \dot{f}\left[B^{\prime} \phi^{\prime}\left(e^{B}-3\right)-2 \phi^{\prime \prime}\left(e^{B}-1\right)\right] \\
4 e^{B}\left(e^{B}-r A^{\prime}-1\right)=-\phi^{\prime 2} r^{2} e^{B}+8\left(e^{B}-3\right) \dot{f} A^{\prime} \phi^{\prime} \\
e^{B}\left[r A^{\prime 2}-2 B^{\prime}+A^{\prime}\left(2-r B^{\prime}\right)+2 r A^{\prime \prime}\right]=-\phi^{\prime 2} r e^{B} \\
+8 \phi^{\prime 2} \ddot{f} A^{\prime}+4 \dot{f}\left[\phi^{\prime}\left(A^{\prime 2}+2 A^{\prime \prime}\right)+A^{\prime}\left(2 \phi^{\prime \prime}-3 B^{\prime} \phi^{\prime}\right)\right]
\end{gathered}
$$

while the scalar equation reads

$$
2 r \phi^{\prime \prime}+\left(4+r A^{\prime}-r B^{\prime}\right) \phi^{\prime}+\frac{4 \dot{f} e^{-B}}{r}\left[\left(e^{B}-3\right) A^{\prime} B^{\prime}-\left(e^{B}-1\right)\left(2 A^{\prime \prime}+A^{\prime 2}\right)\right]=0 .
$$

The unknown quantities, that we seek to determine through the solution of the system of Eqs.

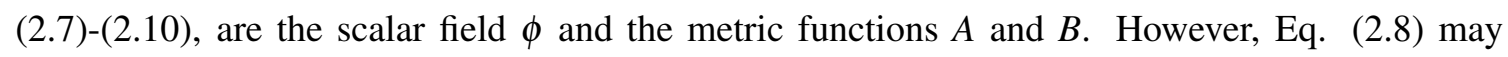
take the form of a second-order polynomial with respect to $e^{B}$, which can then be solved to give $e^{B}=\left(-\beta \pm \sqrt{\beta^{2}-4 \gamma}\right) / 2$, where

$$
\beta=\frac{r^{2} \phi^{\prime 2}}{4}-\left(2 \dot{f} \phi^{\prime}+r\right) A^{\prime}-1, \quad \gamma=6 \dot{f} \phi^{\prime} A^{\prime}
$$

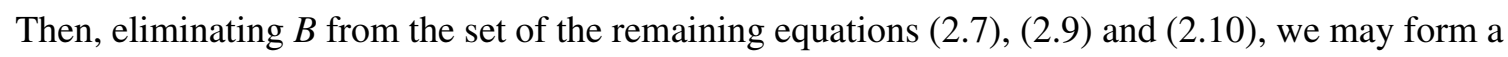
system of two independent, ordinary differential equations of second order for the functions $A$ and $\phi:$

$$
A^{\prime \prime}=\frac{P}{S}, \quad \phi^{\prime \prime}=\frac{Q}{S} .
$$

The functions $P, Q$ and $S$ are rather complicated expressions of $\left(r, \phi^{\prime}, A^{\prime}, \dot{f}, \ddot{f}\right)$ and may be found in [ए]].

We will first study analytically our set of field equations in order to demonstrate that these may allow for the construction of a black-hole solution with a regular horizon provided that the, 
otherwise arbitrary, coupling function $f$ satisfies a set of simple constraints. For a sphericallysymmetric spacetime, the presence of a horizon is realised when

$$
e^{A(r)} \rightarrow 0, \quad e^{-B(r)} \rightarrow 0
$$

In fact, the correct behaviour of $e^{-B}$ follows from the one of $e^{A}$ only for the (+) sign in front of the square-root in the expression for $e^{B}$ that follows from Eq. ([2.8); thus, from now on we focus only on that sign. Also, the regularity of the horizon is guaranteed by the requirement that the scalar field and its derivatives remain finite there. As we will soon prove, the vanishing of $e^{A}$ at the horizon is equivalent to $A^{\prime} \rightarrow \infty$. Therefore, assuming that $A^{\prime} \rightarrow \infty$ while $\phi^{\prime}$ remains finite as $r \rightarrow r_{h}$, Eqs. (2.2]) take the approximate forms

$$
\begin{aligned}
& A^{\prime \prime}=-\frac{r^{4}+4 r^{3} \phi^{\prime} \dot{f}+4 r^{2} \phi^{2} \dot{f}^{2}-24 \dot{f}^{2}}{r^{4}+2 r^{3} \phi^{\prime} \dot{f}-48 \dot{f}^{2}} A^{\prime 2}+\ldots \\
& \phi^{\prime \prime}=-\frac{\left(2 \phi^{\prime} \dot{f}+r\right)\left(r^{3} \phi^{\prime}+12 \dot{f}+2 r^{2} \phi^{\prime 2} \dot{f}\right)}{r^{4}+2 r^{3} \phi^{\prime} \dot{f}-48 \dot{f}^{2}} A^{\prime}+\ldots
\end{aligned}
$$

The second of the above equations leads to the divergence of $\phi^{\prime \prime}$ at the horizon if $f(\phi)$ is either zero or left unconstrained. However, $\phi^{\prime \prime}$ may be rendered finite if either one of the two expressions in the numerator of Eq. (2.5I) is zero close to the horizon.

For the choice $\left(2 \phi^{\prime} \dot{f}+r\right)=0$, and for every elementary form of $f(\phi)$ we have tried, we are always led to either a divergent or a trivial scalar field near the horizon. Thus, for the construction of a regular horizon in the presence of a non-trivial scalar field, we are led to consider the second choice:

$$
r^{3} \phi^{\prime}+12 \dot{f}+2 r^{2} \phi^{\prime 2} \dot{f}=0 .
$$

This may be easily solved to yield:

$$
\phi_{h}^{\prime}=\frac{r_{h}}{4 \dot{f}_{h}}\left(-1 \pm \sqrt{1-\frac{96 \dot{f}_{h}^{2}}{r_{h}^{4}}}\right),
$$

where all quantities have been evaluated at $r_{h}$. To ensure that $\phi_{h}^{\prime}$ is real, the quantity under the square-root should be always positive; therefore, we must impose the following constraint on the coupling function

$$
\dot{f}_{h}^{2}<\frac{r_{h}^{4}}{96}
$$

Going back to the 1st of Eqs. ([2] ), and using the constraint ([.]Z), we find that the coefficient of $A^{\prime 2}$ simplifies to -1 . Then, upon integration with respect to $r$, we obtain $A^{\prime}=\left(r-r_{h}\right)^{-1}+\mathscr{O}(1)$. This is, in fact, in accordance to our earlier claim that $A^{\prime} \rightarrow \infty$ close to the horizon. We may now put everything together and write the near-horizon solution as

$$
\begin{aligned}
& e^{A}=a_{1}\left(r-r_{h}\right)+\ldots \\
& e^{-B}=b_{1}\left(r-r_{h}\right)+\ldots \\
& \phi=\phi_{h}+\phi_{h}^{\prime}\left(r-r_{h}\right)+\phi^{\prime \prime}\left(r-r_{h}\right)^{2}+\ldots
\end{aligned}
$$


The above describes indeed a regular black-hole horizon in the presence of a scalar field provided that $\phi_{h}^{\prime}$ and the coupling function $f$ satisfy the constraints (2.17)-([2.18).

We will now study the regime of asymptotic infinity, to show that a general coupling function $f(\phi)$ for the scalar field does not interfere with the requirement that an asymptotically-flat limit exists for the spacetime ([2.3)). We will assume the following power-law expressions for the metric functions and scalar field, in the limit $r \rightarrow \infty$,

$$
e^{A}=1+\sum_{n=1}^{\infty} \frac{p_{n}}{r}, \quad e^{B}=1+\sum_{n=1}^{\infty} \frac{q_{n}}{r}, \quad \phi=\phi_{\infty}+\sum_{n=1}^{\infty} \frac{d_{n}}{r}
$$

Substituting these expressions into the field equations, we may determine the arbitrary coefficients $\left(p_{n}, q_{n}, d_{n}\right)$. In fact, $p_{1}$ and $d_{1}$ remain arbitrary, and we associate them with the ADM mass and scalar charge, respectively: $p_{1} \equiv-2 M$ and $d_{1}=D$. Then, the asymptotic solutions for the metric functions and scalar field at large distances read:

$$
\begin{aligned}
e^{A} & =1-\frac{2 M}{r}+\frac{M D^{2}}{12 r^{3}}+\frac{24 M D \dot{f}+M^{2} D^{2}}{6 r^{4}}+\ldots, \\
e^{B} & =1+\frac{2 M}{r}+\frac{16 M^{2}-D^{2}}{4 r^{2}}+\frac{32 M^{3}-5 M D^{2}}{4 r^{3}} \\
& +\frac{16 M\left(48 M^{3}-13 M D^{2}-24 D \dot{f}\right)+3 D^{4}}{48 r^{4}}+\ldots, \\
\phi & =\phi_{\infty}+\frac{D}{r}+\frac{M D}{r^{2}}+\frac{32 M^{2} D-D^{3}}{24 r^{3}}+\frac{12 M^{3} D-24 M^{2} \dot{f}-M D^{3}}{6 r^{4}}+\ldots
\end{aligned}
$$

The above asymptotic behaviour is characterised by the ADM mass $M$ and scalar charge $D$ of the black hole. We also note that the explicit form of the coupling function $f(\phi)$ is almost irrelevant as it first makes its appearance in terms of order $\mathscr{O}\left(1 / r^{4}\right)$ in the above expressions. We may therefore conclude that the scalar-tensor theory (R.d) with a general coupling function $f(\phi)$ is always compatible with either a regular horizon or an asymptotically-flat limit.

However, no complete black-hole solution may be constructed unless the aforementioned asymptotic solutions are smoothly matched. To investigate whether this is in principle possible, we turn to the novel no-hair theorem $[8]$ and examine its requirements under which it may forbid the existence of such a solution. This theorem states first that, at asymptotic infinity, the $T_{r}^{r}$ component of the energy-momentum tensor is positive and decreasing. Indeed, using the asymptotic solution $([2.23)$, we find that this has the form

$$
T_{r}^{r}=\frac{e^{-B} \phi^{\prime}}{4}\left[\phi^{\prime}-\frac{8 e^{-B}\left(e^{B}-3\right) \dot{f} A^{\prime}}{r^{2}}\right] \simeq \frac{\phi^{\prime 2}}{4} \sim \mathscr{O}\left(\frac{1}{r^{4}}\right) .
$$

As expected, our result agrees with the one derived in [8] for a minimally-coupled scalar field since at large distances, where the curvature of spacetime is small, we do not expect the quadratic GB term to play any role in the components of the energy-momentum tensor. In the near-horizon regime, the requirements of the novel no-hair theorem dictate that $T_{r}^{r}$ is negative and increasing; if that holds, then the smooth connection of these two asymptotic behaviours for $T_{r}^{r}$ would demand the presence of an extremum at an intermediate point in its profile, a feature that may be excluded 
by using the positivity and conservation of energy density. However, in our case, employing the asymptotic solutions (2.19)-(‥21), we find that

$$
T_{r}^{r}=-\frac{2 e^{-B}}{r^{2}} A^{\prime} \phi^{\prime} \dot{f}+\mathscr{O}\left(r-r_{h}\right)
$$

If we use the fact that close to the horizon, $A^{\prime}>0$, the above means that

$$
\operatorname{sign}\left(T_{r}^{r}\right)_{h}=-\operatorname{sign}\left(\dot{f}_{h} \phi_{h}^{\prime}\right)=1 \mp \sqrt{\left.1-96 \dot{f}^{2} / r_{h}^{4}\right)}>0,
$$

where we have used the constraint Eq. (2.17) for the regularity of the horizon. Thus, the above expression is always positive-definite, and the second clause of the novel no-hair theorem is not true in our case. Also, we find that $T_{r}^{r}$ is always decreasing close to $r_{h}$ and as a result, the novel no-hair theorem is non-applicable in our theory. In other words, the presence of the GB term near the horizon - where the curvature is strong - plays an important role and changes dramatically the profile of the $T_{r}^{r}$, in a way that does not forbid the smooth connection of its two asymptotic behaviours.

In order to complete the analytical study of the theory, let us also address the older version of the no-hair theorem for scalar fields [四], that employs the scalar equation of motion. As we will shortly see, this also fails to exclude the existence of black-hole solutions in our theory (R.T. Multiplying the scalar equation (대) by $f(\phi)$ and integrating over the black-hole exterior region, we obtain the integral constraint

$$
\int d^{4} x \sqrt{-g} f(\phi)\left[\nabla^{2} \phi+\dot{f}(\phi) R_{G B}^{2}\right]=0 .
$$

Integrating by parts the first term, the above becomes

$$
\int d^{4} x \sqrt{-g} \dot{f}(\phi)\left[\partial_{\mu} \phi \partial^{\mu} \phi-f(\phi) R_{G B}^{2}\right]=0 .
$$

The boundary term $\left[\sqrt{-g} f(\phi) \partial^{\mu} \phi\right]_{r_{h}}^{\infty}$ vanishes both at the horizon (due to the $e^{(A-B) / 2}$ factor) and at infinity (due to the $\partial^{\mu} \phi$ factor). Since $\phi=\phi(r)$, the first term in Eq. (L.28) gives $\partial_{\mu} \phi \partial^{\mu} \phi=$ $g^{r r}\left(\partial_{r} \phi\right)^{2}>0$ throughout the exterior region. Also, for the metric ([2.3), the GB term has the explicit form

$$
R_{G B}^{2}=\frac{2 e^{-2 B}}{r^{2}}\left[\left(e^{B}-3\right) A^{\prime} B^{\prime}-\left(e^{B}-1\right)\left(2 A^{\prime \prime}+A^{\prime 2}\right)\right] .
$$

Employing the asymptotic solutions near the horizon ([.19)-(․20) and at infinity ([2.23), we may easily find the results

$$
\left.R_{G B}^{2}\right|_{r_{h}}=\frac{12 e^{-2 B}}{r^{4}} A^{\prime 2}+\cdots,\left.\quad R_{G B}^{2}\right|_{\infty}=\frac{48 M^{2}}{r^{6}}+\cdots
$$

Both of the above expressions are positive definite. Therefore, in the simplest possible case where $R_{G B}^{2}$ retains a positive sign throughout the radial regime (as we will indeed find) and $f(\phi)$ is also sign-definite, Eq. (2.28) allows for black-hole solutions with scalar hair for every choice of the coupling function that merely satisfies $f(\phi)>0$. We thus observe that the old no-hair theorem seems to be more easily evaded for the theory (2.1) than the novel one under a simple assumption on the sign of the scalar-field-GB coupling function. 

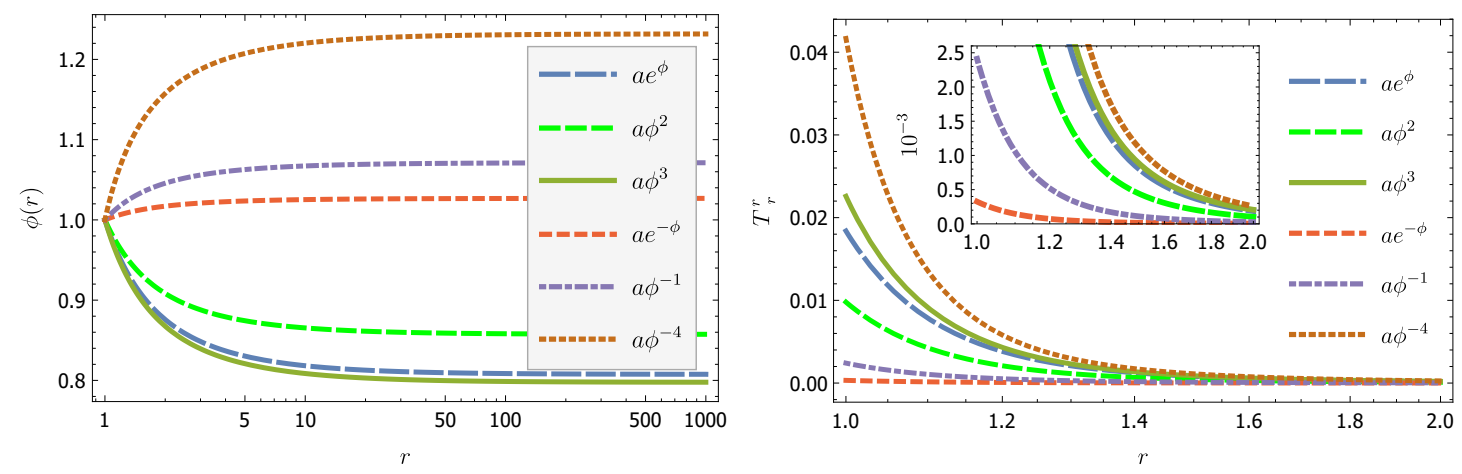

Figure 1: The scalar field $\phi$ (left plot) and the $T_{r}^{r}$ component (right plot) for different coupling functions $f(\phi)$, for $a=0.01$ and $\phi_{h}=1$ [प्प].

The above results and theoretical arguments clearly open the way for the construction of novel black-hole solutions in the context of the general theory (R.D). We have therefore numerically integrated the system of equations (2.12), and determined a large number of black-hole solutions with scalar hair for a variety of forms of the coupling function $f(\phi)$ : exponential, odd and even power-law, odd and even inverse-power-law. These forms are all simple, natural choices to keep the GB term in the 4-dimensional theory. Once the form of $f(\phi)$ was chosen, we only needed to decide the input values $\left(\phi_{h}, \phi_{h}^{\prime}\right)$. The first quantity was in fact a free parameter constrained only by the condition (2.18) while the second one was uniquely determined by the regularity constraint ([2.]7) of the black-hole horizon. In fact, following this "recipe", every pair of initial values $\left(\phi_{h}, \phi_{h}^{\prime}\right)$ leads to a regular black-hole solution with a non-trivial scalar hair.

At the left plot of Fig. 四, we depict the profile of the scalar field for an indicative set of those solutions. For easy comparison, the coupling constant has been set to $a=0.01$ and the near-horizon value of the field to $\phi_{h}=1$ in all cases. The profiles of the scalar field are everywhere finite exhibiting either a decreasing or increasing behaviour away from the black-hole horizon. This latter feature is dictated by the constraint (2.17) that demands $\phi_{h}^{\prime} \dot{f}_{h}<0$. Therefore, for $f(\phi)=\left(a e^{\phi}, a \phi^{2}, a \phi^{3}\right)$, that all have $\dot{f}_{h}>0$, the scalar field must have a negative $\phi_{h}^{\prime}$; for $f(\phi)=\left(a e^{-\phi}, a \phi^{-1}, a \phi^{-4}\right)$, that have $\dot{f}_{h}<0$, Eq. (․ㅣ) demands a positive $\phi_{h}^{\prime}$. In all cases, the scalar field reduces to a constant asymptotic value at large distances from the black-hole horizon.

At the right plot of Fig. 四, we present the $T_{r}^{r}$ component of the energy-momentum tensor for the same indicative family of solutions. Again, $T_{r}^{r}$ remains finite and exhibits the features that guaranteed the evasion of the novel no-hair theorem: it is always positive and decreasing both near the black-hole horizon and far away from it. It is worth mentioning that the decrease of $T_{r}^{r}$ close to the horizon - a feature that could not be ensured as it involved $\phi_{h}^{\prime \prime}$ which is not an input parameter - automatically appears for every solution we have found, and does not need any further action or fine-tuning of the free parameters. We finally note that, for $\phi>0$, all the above forms of $f(\phi)$ satisfy also the constraint $f(\phi)>0$, derived above for the evasion of the old no-hair theorem.

We also present the solution for the two metric components $\left(\left|g_{t t}\right|, g_{r r}\right)$ and the GB term $R_{G B}^{2}$ at the left and right plots of Fig. $\square$, respectively, for the indicative case of $f(\phi)=a e^{-\phi}$. The metric components exhibit the expected behaviour near the black-hole horizon with $g_{t t}$ vanishing and $g_{r r}$ diverging at $r_{h}=1$ (where we have fixed the horizon radius). In order to ensure asymptotic flatness 

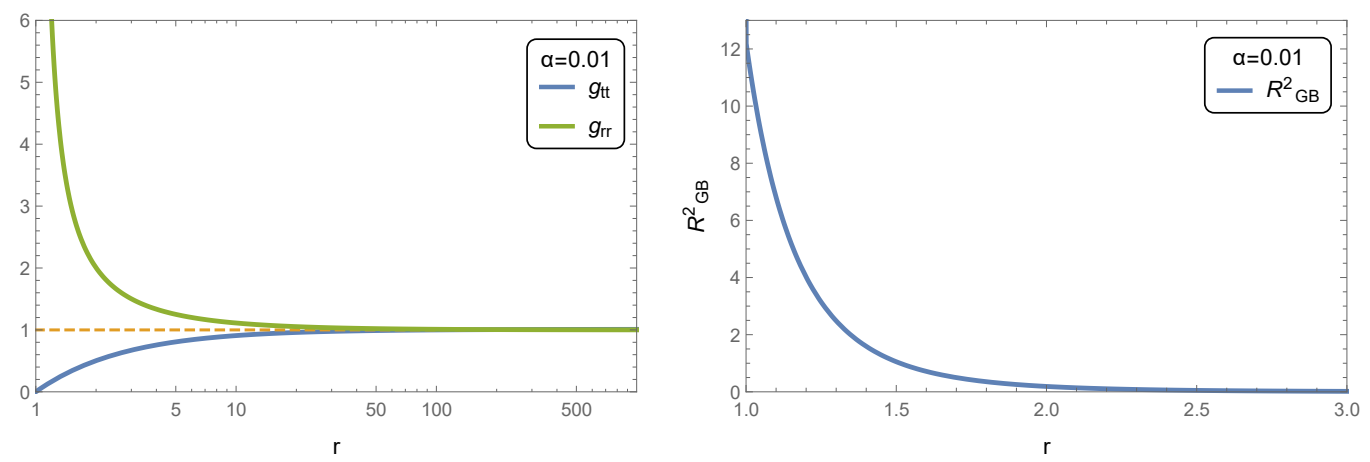

Figure 2: The metric components (left plot) and the Gauss-Bonnet term $R_{G B}^{2}$ (right plot) for the exponential coupling function $f(\phi)=a e^{-\phi}$, for $a=0.01$ and $\phi_{h}=1$ [एव].

at radial infinity, the free parameter $a_{1}$ appearing in the near-horizon solution (R.CQ) is appropriately chosen. On the other hand, the GB term remains finite and positive-definite over the entire radial domain - in fact it displays the monotonic behaviour, hinted by its two asymptotic limits (2.30), that causes the evasion of the old no-hair theorem. As expected, it contributes significantly near the horizon, where the curvature is large, and quickly fades away as we move towards larger distances. The profile of the metric components and GB term exhibit the same qualitative behaviour in all families of black-hole solutions that we have found.

Some of the characteristics of the black-hole solutions we found [19] are represented in the two plots of Fig. [3, where we depict the indicative case of $f(\phi)=a / \phi$. The scalar charge $D$ depicted in the left plot, that characterises the scalar field at infinity, is in fact a function of the black-hole mass. The determination of an analytic relation that connects $D$ with $M$ is not an easy task (for the exponential case, that task was performed in [ㅁ]]). Nevertheless, a dependence of $D$ on $M$ always emerged through the numerical integration, for every form of the coupling function, a result that clearly renders the scalar hair secondary. Also, a common characteristic in all cases is that, as the mass of the black hole increases, the scalar charge decreases and eventually vanishes when our black-hole solutions matches the Schwarzschild solution.
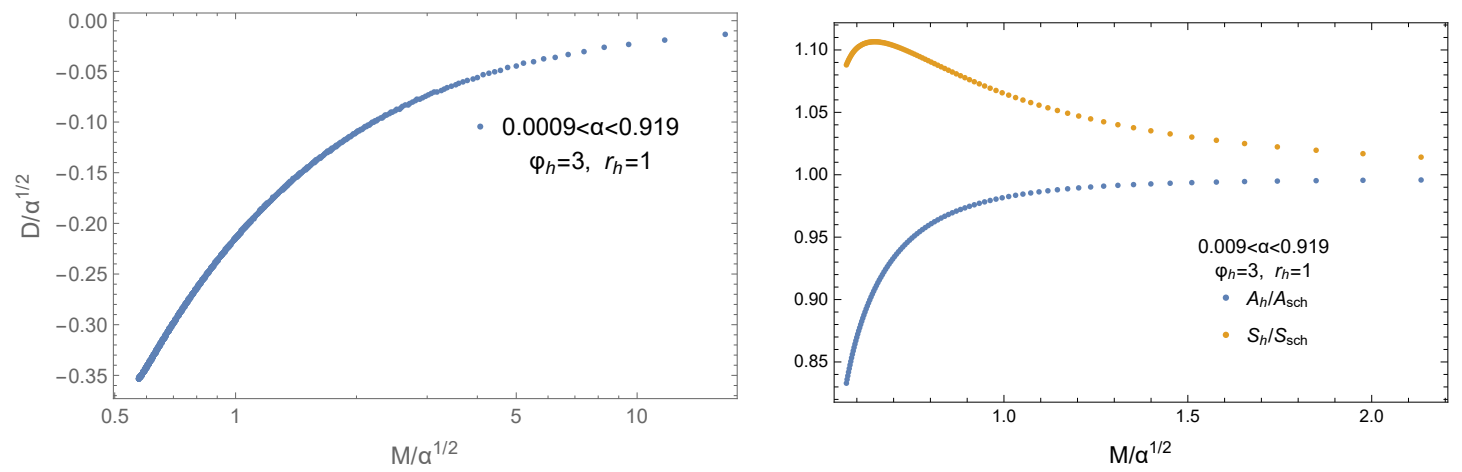

Figure 3: The scalar charge $D$ (left plot), and the ratios $A_{h} / A_{S c h}$ and $S_{h} / S_{S c h}$ (right plot, lower and upper curve respectively) in terms of the mass $M$, for $f(\phi)=a / \phi$ [ए]]. 
The horizon area of the black hole, given by $A_{h}=4 \pi r_{h}^{2}$, is depicted in the right plot of Fig. [] normalised in units of the horizon area of the Schwarzschild solution with the same mass. We observe that this ratio is always smaller than unity, which means that all GB black-hole solutions are smaller than their GR analogues. The area curve also stops abruptly at its lower end, thus exhibiting the existence of a lower bound on the horizon area and thus on the mass of the black hole (see also [12, [25]). Beyond this lower value, the black hole ceases to exist - the latter feature is due to the bound (2.18) emerging from the positivity of the quantity under the square-root in Eq. ([.]Z), that may be also written as $r_{h}^{2}>4 \sqrt{6}\left|\dot{f}_{h}\right|$. Finally, the entropy of the black-hole solutions may be computed, for an arbitrary form of $f(\phi)$, following the method outlined in [114]. Then, it is found to be

$$
S_{h}=\frac{A_{h}}{4}+4 \pi f\left(\phi_{h}\right) .
$$

The ratio of the entropy $S_{h}$ of the GB black holes over the one of the Schwarzschild black hole, $S_{S c h}=A_{h} / 4$, is also depicted in the right plot of Fig. B. For the particular form of the coupling function employed in this case, i.e. the inverse linear form, the entropy ratio comes out to be larger than unity for the entire mass range, a result that renders this particular family of solutions more thermodynamically stable than the corresponding Schwarzschild black hole. For different forms of the coupling function though, the curve of the entropy ratio may lie in whole or in parts below unity thus revealing an instability for the entire or parts of the mass regime. In all cases, as the mass $M$ increases, the entropy ratio always reduces to unity as the Schwarzschild limit is approached.

\section{Asymptotically (Anti)-de Sitter GB black holes}

We would like now to extend the previous study by adding a cosmological constant $\Lambda$ in the spacetime, either positive or negative. The action functional of the theory then reads [ [D]]

$$
S=\frac{1}{16 \pi} \int d^{4} x \sqrt{-g}\left[R-\frac{1}{2} \partial_{\mu} \phi \partial^{\mu} \phi+f(\phi) R_{G B}^{2}-2 \Lambda\right] .
$$

In this case, the field equations (2.4) remain unchanged apart from the shift

$$
T_{\mu v} \rightarrow T_{\mu v}-\Lambda g_{\mu v}
$$

due to the addition of the cosmological constant. Despite the minimal change, though, the situation differs a lot from the one where the cosmological constant is ignored. First of all, we do not expect the spacetime to be asymptotically-flat any more - we expect that this may have the form of an asymptotically (Anti)-de Sitter spacetime. However, this will also depend on the behaviour of the scalar field itself since this also contributes to the form of the energy-momentum tensor at large distances from the black-hole spacetime. In addition, since the cosmological constant permeates the whole spacetime, we expect that its presence would be important also close to the black-hole horizon, and that the previous constraints, under which regular black-hole solutions have emerged, may be now modified.

To investigate the above, we repeat the analytical study of the field equations both close to and far away from the black-hole horizon. In order to determine first the number of independent 
unknown functions of the problem, we use again the $(r r)$-component of the gravitational equations to solve for the metric function $e^{B}$ in terms of $A$ and $\phi$. In this case, we find

$$
e^{B}=\frac{-\beta \pm \sqrt{\beta^{2}-4 \alpha \gamma}}{2 \alpha}
$$

where now

$$
\alpha=1-\Lambda r^{2}, \quad \beta=\frac{r^{2} \phi^{\prime 2}}{4}-\left(2 \dot{f} \phi^{\prime}+r\right) A^{\prime}-1, \quad \gamma=6 \dot{f} \phi^{\prime} A^{\prime} .
$$

Employing the above into the remaining field equations, we obtain again a system of two independent, ordinary, 2nd order differential equations for $A$ and $\phi$ similar to the one given by Eq. (L.J2) the interested reader may find the new expressions of the quantities $P, Q$ and $S$ in [ [DU].

Close to the horizon, we demand again that $A^{\prime} \rightarrow \infty$ while the scalar field and its derivatives remain finite. Then, the system (2.J2) demands once again that the following constraint should hold [DOD]

$$
\phi_{h}^{\prime}=-\frac{r_{h}^{3}\left(1-\Lambda r_{h}^{2}\right)+16 \Lambda r_{h} \dot{f}_{h}^{2}\left(3-\Lambda r_{h}^{2}\right) \pm\left(1-\Lambda r_{h}^{2}\right) \sqrt{C}}{4 \dot{f}\left[r_{h}^{2}-\Lambda\left(r_{h}^{4}-16 \dot{f}_{h}^{2}\right)\right]},
$$

where all quantities have been evaluated at $r=r_{h}$. The quantity $C$ under the square root stands for the following combination

$$
C=256 \Lambda \dot{f}_{h}^{4}\left(\Lambda r_{h}^{2}-6\right)+32 r_{h}^{2} \dot{f}_{h}^{2}\left(2 \Lambda r_{h}^{2}-3\right)+r_{h}^{6} \geq 0,
$$

and must always be non-negative for $\phi_{h}^{\prime}$ to be real. Using again the constraint (B.5) in the approximated form of the first of Eqs. ([2] ) for $A$ close to the horizon, we may uniquely construct the form of the black-hole horizon solution. This has exactly the same functional form as the one constructed for the case of vanishing $\Lambda$, i.e. Eqs. (‥19)-([2.21). The presence of the cosmological constant modifies though the exact expressions of the basic constraint (3.5) for $\phi_{h}^{\prime}$ and of the quantity $C$ given in (B.6), the validity of which ensures the existence of a regular black-hole horizon. As in the case of a vanishing cosmological constant, the exact form of the coupling function $f(\phi)$ does not affect the emergence of an asymptotic, regular black-hole solution.

At large distances from the black-hole horizon, we must distinguish between a positive and a negative cosmological constant. In the presence of a positive cosmological constant, a second horizon, the cosmological one, is expected to emerge at a radial distance $r=r_{c}>r_{h}$. We demand that this horizon is also regular, with the scalar field $\phi$ and its derivatives being again finite in its vicinity. By following a method identical to the one followed in section 2 near the black-hole horizon, we arrive at the following constraint

$$
\phi_{c}^{\prime}=-\frac{r_{c}^{3}\left(1-\Lambda r_{c}^{2}\right)+16 \Lambda r_{c} \dot{f}_{c}^{2}\left(3-\Lambda r_{c}^{2}\right) \pm\left(1-\Lambda r_{c}^{2}\right) \sqrt{\tilde{C}}}{4 \dot{f}\left[r_{c}^{2}-\Lambda\left(r_{c}^{4}-16 \dot{f}_{c}^{2}\right)\right]}
$$

for $\phi^{\prime}$ at the cosmological horizon, with $\tilde{C}$ now being given by the non-negative expression

$$
\tilde{C}=256 \Lambda \dot{f}_{c}^{4}\left(\Lambda r_{c}^{2}-6\right)+32 r_{c}^{2} \dot{f}_{c}^{2}\left(2 \Lambda r_{c}^{2}-3\right)+r_{c}^{6} \geq 0 .
$$


Employing Eq. (3.]), the solution for the metric function $A$ may be again constructed. Overall, the asymptotic solution of the field equations near a regular, cosmological horizon will have the form

$$
\begin{aligned}
e^{A} & =a_{2}\left(r_{c}-r\right)+\ldots, \\
e^{-B} & =b_{2}\left(r_{c}-r\right)+\ldots, \\
\phi & =\phi_{c}+\phi_{c}^{\prime}\left(r_{c}-r\right)+\phi_{c}^{\prime \prime}\left(r_{c}-r\right)^{2}+\ldots,
\end{aligned}
$$

where care has been taken for the fact that $r \leq r_{c}$. Once again, the explicit form of the coupling function $f(\phi)$ is of minor importance for the existence of a regular, cosmological horizon.

For a negative cosmological constant, and at large distances from the black-hole horizon, we expect the spacetime to assume a form close to that of the Schwarzschild-Anti-de Sitter solution. Thus, we assume the following approximate forms for the metric functions

$$
\begin{aligned}
e^{A(r)} & =\left(k-\frac{2 M}{r}-\frac{\Lambda_{\text {eff }}}{3} r^{2}+\frac{q_{2}}{r^{2}}\right)\left(1+\frac{q_{1}}{r^{2}}\right)^{2}, \\
e^{-B(r)} & =k-\frac{2 M}{r}-\frac{\Lambda_{e f f}}{3} r^{2}+\frac{q_{2}}{r^{2}},
\end{aligned}
$$

where $k, M, \Lambda_{e f f}$ and $q_{1,2}$ are arbitrary constants. Substituting the above expressions into the scalar field equation ([.]d), we obtain at first order the constraint

$$
\phi^{\prime \prime}(r)+\frac{4}{r} \phi^{\prime}(r)-\frac{8 \Lambda_{e f f} \dot{f}}{r^{2}}=0 .
$$

Contrary to what happens close to the horizons (either black-hole or cosmological ones), the form of the coupling function $f(\phi)$ now affects the asymptotic form of the scalar field at large distances. The easiest case is that of a linear coupling function, $f(\phi)=a \phi$, first studied in [26]. The scalar field, at large distances, may then be shown to have the approximate form

$$
\phi(r)=\phi_{\infty}+d_{1} \ln r+\frac{d_{2}}{r^{2}}+\frac{d_{3}}{r^{3}}+\ldots,
$$

where again $\left(\phi_{\infty}, d_{1}, d_{2}, d_{3}\right)$ are arbitrary constant coefficients. In fact, this form describes the scalar field at large distances in the perturbative limit, i.e. in the limit of small GB coupling constant $a$, independently of the exact form of the coupling function. In addition, as our exact numerical analysis has shown, the dominant term in the expression of $\phi$ at large distances from the black-hole horizon is indeed described by a logarithmic term even in the non-perturbative limit. The constant coefficients $d_{1}$ and $\Lambda_{\text {eff }}$ may be determined through the first-order constraints that follow from the gravitational equations, upon substitution of the approximate forms (B.D2)-(B.13), and they are given by

$$
d_{1}=\frac{8}{3} \alpha \Lambda_{e f f}, \quad \Lambda_{e f f}\left(3+\frac{80 \alpha^{2} \Lambda_{e f f}^{2}}{9}\right)=3 \Lambda .
$$

In order to determine the values of the remaining coefficients, one needs to derive higher-order constraints. For example, the coefficients $k, q_{1}$ and $d_{2}$ are found at third-order approximation while 

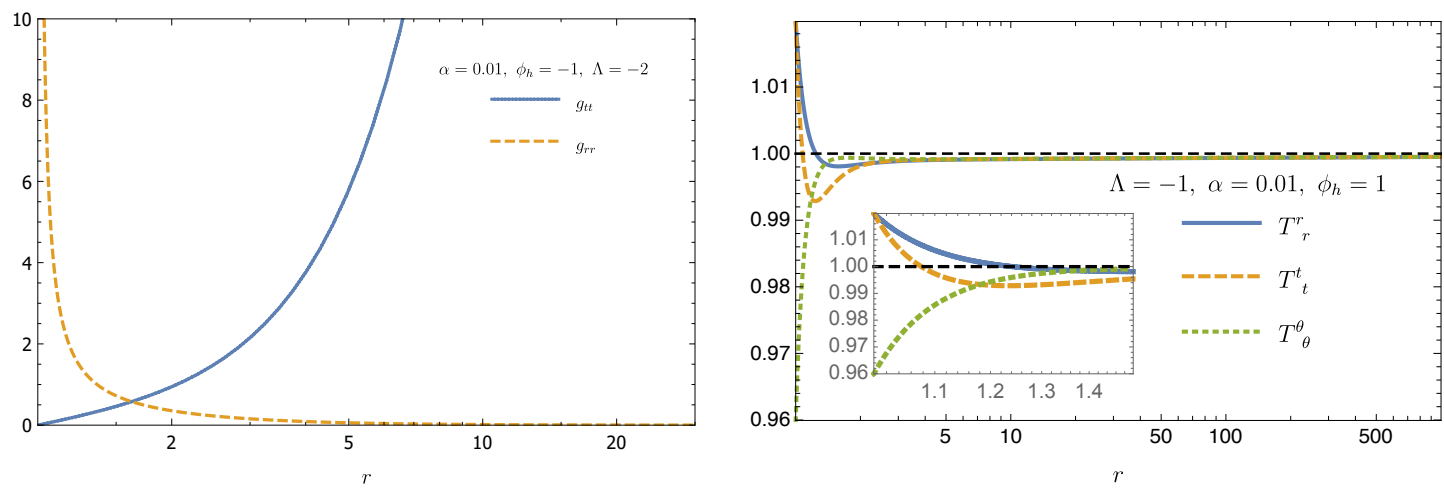

Figure 4: The metric components $\left|g_{t t}\right|$ and $g_{r r}$ (left plot), and the energy-momentum tensor components (right plot) in terms of the radial coordinate $r$, for $f(\phi)=a \phi^{2}$ [20]].

for $q_{2}$ or $d_{3}$ one needs to go even higher. In contrast, the coefficient $M$ remains arbitrary and may be interpreted as the gravitational mass of the solution.

The complete black-hole solutions were determined once again though the numerical integration of the system ([.12). Starting from the case of a negative cosmological constant $(\Lambda<0)$, the analysis proceeded as in the asymptotically-flat case, with regular, black-hole solutions with nontrivial scalar hair and an asymptotically Anti-de Sitter behaviour emerging for every set of input values $\left(\phi_{h}, \phi_{h}^{\prime}\right)$ that satisfy the constraints (B.5) and (B.6). In the left plot of Fig. W, we depict the metric components $\left|g_{t t}\right|$ and $g_{r r}$ for the indicative case of $f(\phi)=a \phi^{2}$. The expected behaviour near the black-hole horizon soon changes to the asymptotic AdS behaviour with the $\left|g_{t t}\right|$ component diverging and the $g_{r r}$ component vanishing at large distances from the black-hole horizon. This behaviour is fully justified by the profiles of the energy-momentum components depicted at the right plot of Fig. 团; we observe that all components reduce to constant values proportional to the negative cosmological constant, and that the scalar field has a "localised" contribution to the energy-momentum tensor of the theory that vanishes at large distances.

The profile of the scalar field for a variety of values of the cosmological constant, and $f(\phi)=$ $a \phi^{2}$ is presented at the left plot of Fig. [1, while the behaviour of the constant coefficient $d_{1}$ for a variety of forms of $f(\phi)$ is depicted at the right plot of the same figure. We observe that, for small values of $\Lambda$, the scalar field approaches an almost constant value at infinity similarly to the asymptotically-flat case. However, as $\Lambda$ increases (in absolute value), the profile of the scalar field changes adopting a non-constant, logarithmic dependence on the radial coordinate. Similar results were derived for all other black-hole solutions produced for the choices $f(\phi)=$ $e^{ \pm \phi}, \phi^{ \pm 2 n}, \phi^{ \pm(2 n+1)}, \ln \phi$. The values of $d_{1}$, at the right plot, give a measure of how much the scalar field deviates from a constant asymptotic value: it is clear that this deviation is larger for the smallmass GB black holes while, as $M$ increases, this coefficient decreases and eventually vanishes when the Schwarzschild-Anti-de Sitter limit is recovered.

We finally present two plots, for the horizon area ratio (left plot) and entropy ratio (right plot) in Fig. 6, again for a variety of black-hole solutions and for different forms of the coupling function $f(\phi)$. We have considered a rather large value of the cosmological constant in order to maximize its effect on these two quantities. We observe that, even for large values of $\Lambda$, the area 

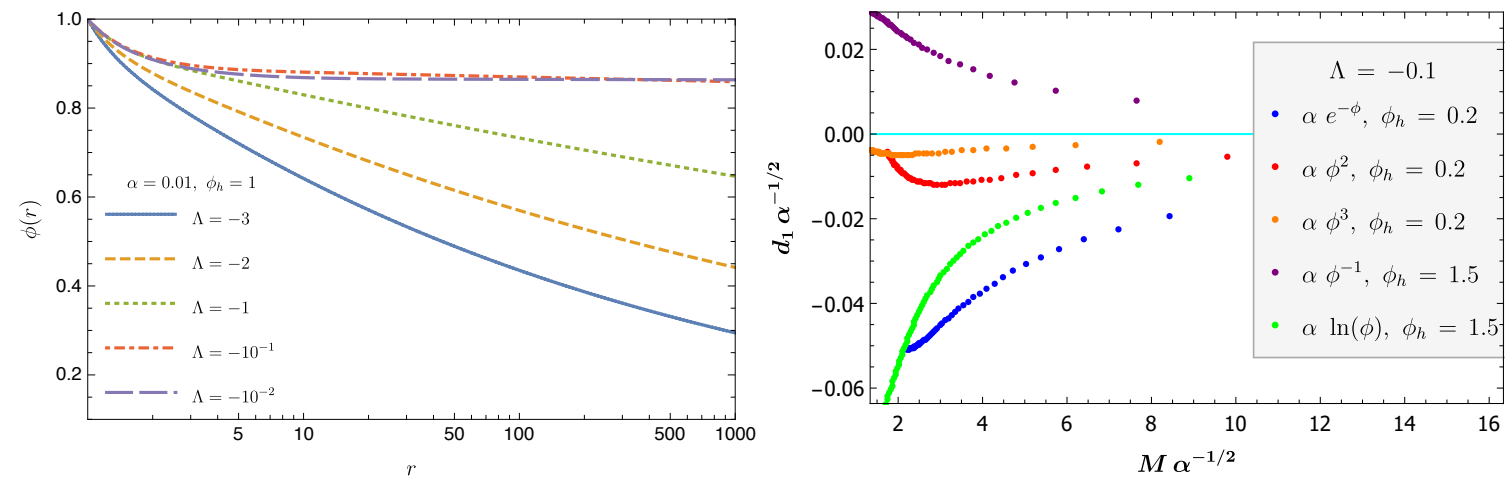

Figure 5: The scalar field $\phi$ (left plot) for a variety of values of the cosmological constant, and the constant coefficient $d_{1}$ (right plot) for a variety of forms of the coupling function $f(\phi)$ [20].

ratio remains smaller than unity (apart from the logarithmic case) - in fact, the black-hole solutions we have found tend to have a larger lower limit for the black-hole horizon radius as $\Lambda$ increases. In addition, the increase in the value of the cosmological constant tends to increase the entropy ratio [with the entropy of the GB black hole being given by the same formula (2.3W)] and therefore to make the GB black holes more thermodynamically stable compared to their SAdS analogues of General Relativity.

Let us now turn to the case of a positive cosmological constant $(\Lambda>0)$. Despite our efforts, no complete black-hole, asymptotically de Sitter solutions were found. Starting from the near-horizon asymptotic solutions (2.19)-(2.21), the numerical integration would proceed as normal but would abruptly stop before the cosmological-horizon solution (B.9)-(B.W) could be reached. Although the two asymptotic solutions near the black-hole and cosmological horizons do independently emerge, the effort to match them in a smooth way via an intermediate solution fails for all choices of parameters we have tried. To demonstrate this, in Fig. $\square$ we display the result of our numerical integration for the indicative case of $\alpha=0.01, \phi_{h}=-1$ and $\Lambda=0.01$. The coupling function has been chosen to be $f(\phi)=\alpha e^{-\phi}$, however, the same qualitative behaviour was found for every choice of $f(\phi)$ we have considered. From the metric functions and the scalar-field profiles displayed in the two
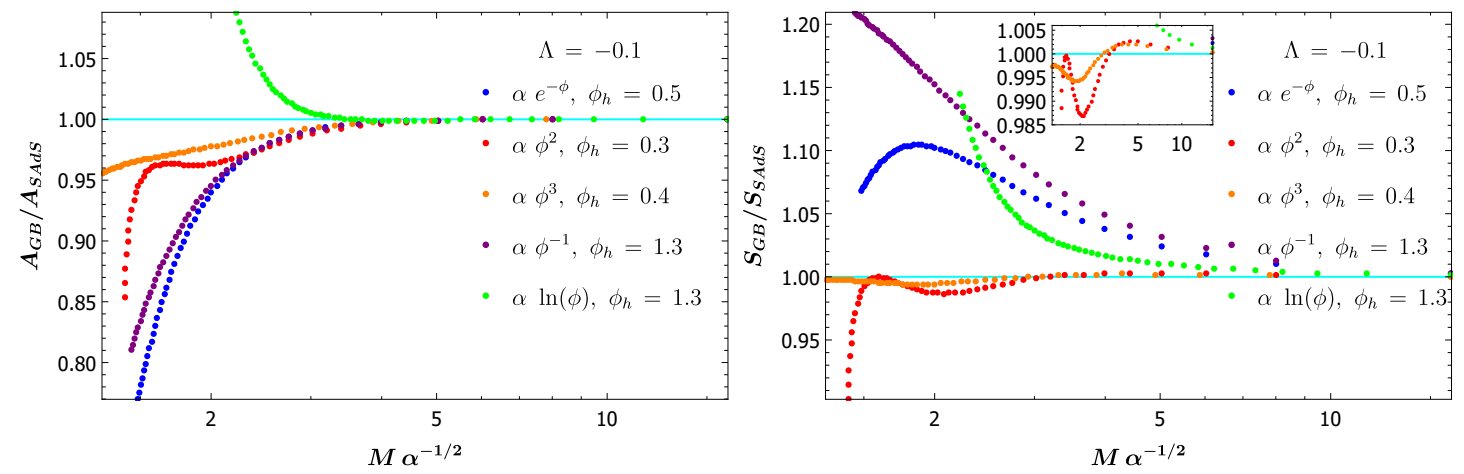

Figure 6: The horizon area $A_{G B} / A_{S A d S}$ (left plot) and entropy ratio $S_{G B} / S_{S A d S}$ (right plot) for a variety of forms of the coupling function $f(\phi)$ and in terms of the mass $\mathrm{M}$ [ㅁ]]. 

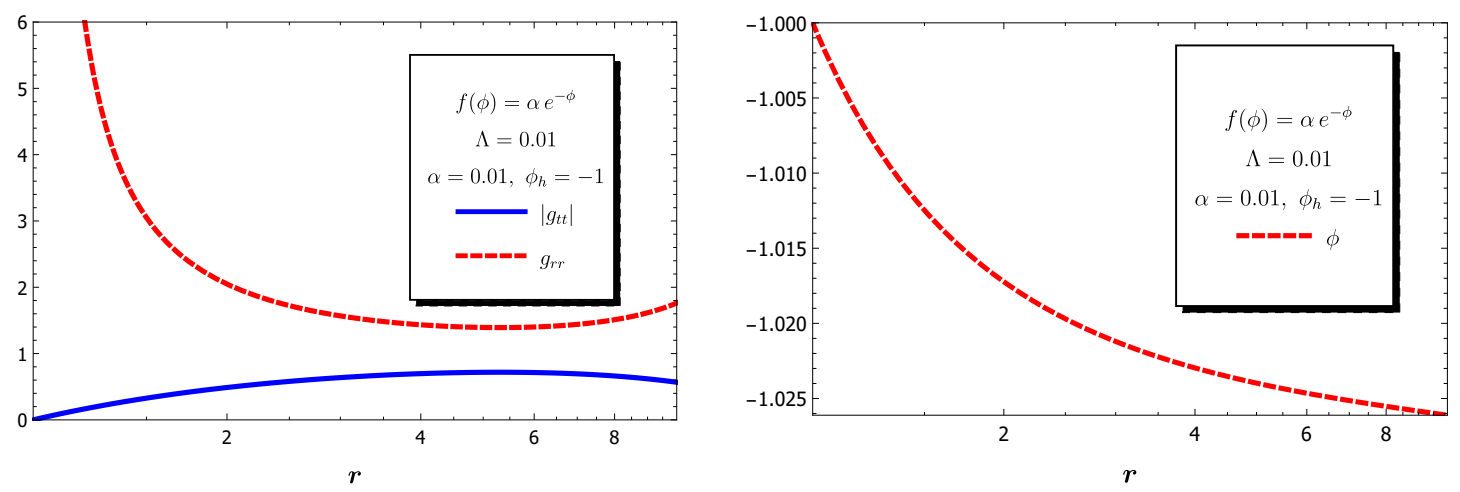

Figure 7: The metric functions $\left|g_{t t}\right|$ and $g_{r r}$ of the spacetime (left plot) and the scalar field $\phi$ (right plot) in terms of the radial coordinate $r$, for a positive cosmological constant and coupling function $f(\phi)=\alpha e^{-\phi}$ [ए]].

plots, we clearly see that an asymptotic solution describing a regular black-hole horizon is indeed formed but the cosmological horizon solution is never reached.

Although it is very difficult to generalise the no-hair analysis presented in the previous section, one could, nevertheless, gain some understanding of the situation by examining the form of the near-horizon value of the $T_{r}^{r}$ component of the energy-momentum tensor given that is given by

$$
T_{r}^{r}=-\frac{2 e^{-B}}{r^{2}} A^{\prime} \phi^{\prime} \dot{f}-\Lambda+\mathscr{O}\left(r-r_{h}\right) .
$$

It becomes clear that the presence of a negative cosmological constant $(\Lambda<0)$ in the theory always gives a positive contribution to $T_{r}^{r}$, and enhances the probability of obtaining regular black holes. On the other hand, the contribution of a positive cosmological constant $(\Lambda>0)$ to $T_{r}^{r}$ is always negative, and this makes the evasion of the no-hair theorem less likely.

\section{The pure scalar-Gauss-Bonnet theory}

As we saw in Section 2, where the asymptotically-flat case was studied, the quadratic GB term is negligible at large distances from the black-hole horizon but is very important near the horizon in fact, the smaller the black hole, the larger the curvature, and the more important the GB term is. Can we then claim that there is a class of black-hole solutions that may be attributed almost solely to the GB term?

The motivation for this may be looked for in the cosmological set-up. We mentioned previously that, in the context of the moduli part of the heterotic superstring effective action, singularityfree cosmological solutions were found in [23] (and further studied in [27, [28]). These solutions were found numerically due to the complexity of the set of field equations. However, in [RQ], it was demonstrated that, in the early universe, as $t \rightarrow 0$, the Ricci term $R$ becomes negligible while the GB term $R_{G B}^{2}$ dominates. In that limit, the $R$ term may be ignored, upon which the field equations are greatly simplified and thus may be analytically solved. This analysis has yielded, as expected, the class of singularity-free solutions in an analytical form that were smoothly connected to the numerical solutions found in [27]. 
We will therefore investigate whether a regular black-hole solution can arise in the context of a pure scalar-GB theory, i.e. in the absence of the linear Ricci term. By ignoring all terms in the field equations related to the Ricci term, these are simplified - but can we construct again a regular horizon? If we assume as before that, as $r \rightarrow r_{h}, \phi^{\prime}$ remains finite while $A^{\prime}$ diverges, Eq. ([2.8) now yields: $e^{B} \simeq 3+\mathscr{O}\left(1 / A^{\prime}\right)$; but this does not describe a black hole. We may alternatively demand that $e^{B} \rightarrow \infty$ instead, as $r \rightarrow r_{h}$; then, Eq. (2.8) gives: $A^{\prime} \simeq r^{2} \phi^{\prime} / 8 \dot{f}+\mathscr{O}\left(e^{-B}\right)$. In this case, $A(r)$ is the dependent quantity, and Eqs. ([2.7) and (2.9) form a system of two differential equations for $B$ and $\phi$. In the limit $r \rightarrow r_{h}$, we find the results [प्प]

$$
B^{\prime}=-\frac{2}{r} e^{B}+\mathscr{O}\left(e^{-B}\right), \quad \phi^{\prime \prime}=-\frac{e^{B}}{r} \phi^{\prime}+\mathscr{O}\left(e^{-B}\right) .
$$

Upon integration, the first equation leads to the solution $e^{-B}=2 \ln \left(r / r_{h}\right)$, which does resemble a horizon, but the second one reveals that this horizon is not regular unless $\phi^{\prime}\left(r_{h}\right)=0$, an assumption that trivialises the contribution of the GB term.

Alternative ansatzes for the form of the spacetime around the sought-for black hole have also failed to lead to a regular horizon in the absence of the Ricci scalar [34]. A similar negative result was also found in the case where a cosmological constant, either positive or negative, was added to the model. Although, it seems as, in the absence of the Ricci scalar, the GB term may support a non-trivial scalar field, and that in turn the scalar field may keep the GB term in the theory, a pure scalar-GB solution cannot emerge. It is quite likely that the regime of dominance of the GB term over the Ricci term lies beyond the minimum-mass limit, whereas no such "barrier" exists in the cosmological set-up where one can approach an early enough time period, and thus a period of strong enough curvature for the GB term to dominate.

\section{Conclusions}

In the absence of a Quantum Theory of Gravity, the formulation of a generalised theory of gravity may be the way forward in gravitational physics. The Einstein-scalar-Gauss-Bonnet theory is a representative example of such a theory and has been intensively studied for decades leading to novel gravitational and cosmological solutions.

Here, we have considered a general Einstein-scalar-GB theory, and we have demonstrated that the emergence of regular black-hole solutions is a generic feature. For an arbitrary coupling function $f(\phi)$, we were always able to construct a regular black-hole horizon as well as an asymptotically-flat solution at infinity. Under a mild only constraint on the value of $\phi_{h}^{\prime}$, connected to the regularity of the horizon, we explicitly showed that both the old and the novel no-hair theorems are easily evaded. Our numerical analysis has subsequently led to a large number of regular black-hole solutions for different choices of $f(\phi)$, all characterised by a non-trivial scalar hair (for similar black-hole solutions, see also [30, 13] ]).

All GB black-hole solutions are characterised by the existence of a lower bound for the horizon radius of the black hole, and thus a lower-bound on their mass, a feature that distinguishes them from their GR analogues [10, [2]]. Thus, in the small-mass limit, observable effects may include deviations from standard GR in the calculation of the bending angle of light, the precession observed in near-horizon orbits and the spectrum from their accretion discs [32]. The emission 
of scalar radiation strongly depends to the existing coupling of the scalar field to ordinary matter, while the measurement of the characteristic frequencies of the quasi-normal modes will also help to distinguish these solutions from their GR analogues [B3]. Finally, the detection of gravitational waves from black-hole or neutron-star mergers may also help to impose bounds on the parameters of the theory provided that the scalar charge is significant and their physical distance is small.

Subsequently, we have attempted to find black-hole solutions in the presence of a cosmological constant, either positive or negative, in the context of the same theory. Once again, we were able to construct regular asymptotic solutions that described a black-hole horizon at some small radial distance and either a cosmological horizon or an asymptotic Schwarzschild-Anti-de Sitter spacetime at large distances for a positive or negative $\Lambda$, respectively. However, we were able to construct complete black-hole solutions only in the case of a negative cosmological constant, and with the same easiness, for any form of the coupling function $f(\phi)$, as in the asymptotically-flat case. Unfortunately, no black-hole solutions were found for the case of a positive cosmological constant, a case that is of immediate relevance for our 4-dimensional universe. For some yet undetermined reason, the presence of a positive cosmological constant destroys the synergy between the Ricci scalar, the scalar field and the GB term that leads to the emergence of the black-hole solutions.

The latter synergy was confirmed by the analysis that aimed at determining black-hole solutions in the pure scalar-GB theory, i.e. in the absence of the Ricci scalar. Despite our persistent efforts no such solutions were found. The failure to obtain the desired type of solutions clearly demonstrates that the presence of the GB term in the theory is a necessary condition for the emergence of novel black holes but not a sufficient one as it must be supplemented by the presence of the linear Ricci term.

\section{Acknowledgments}

This research is implemented through the Operational Program "Human Resources Development, Education and Lifelong Learning" and is co-financed by the European Union (European Social Fund) and Greek national funds.

\section{References}

[1] R. R. Metsaev and A. A. Tseytlin, Nucl. Phys. B 293 (1987) 385.

[2] D. Lovelock, J. Math. Phys. 12 (1971) 498.

[3] G. W. Horndeski, Int. J. Theor. Phys. 10 (1974) 363.

[4] J. D. Bekenstein, Phys. Rev. Lett. 28 (1972) 452; C. Teitelboim, Lett. Nuovo Cim. 3S2 (1972) 397.

[5] M. S. Volkov and D. V. Galtsov, JETP Lett. 50 (1989) 346; P. Bizon, Phys. Rev. Lett. 64 (1990) 2844; B. R. Greene, S. D. Mathur and C. M. O’Neill, Phys. Rev. D 47 (1993) 2242; K. I. Maeda, T. Tachizawa, T. Torii and T. Maki, Phys. Rev. Lett. 72 (1994) 450.

[6] H. Luckock and I. Moss, Phys. Lett. B 176 (1986) 341; S. Droz, M. Heusler and N. Straumann, Phys. Lett. B 268 (1991) 371.

[7] J. D. Bekenstein, Annals Phys. 82 (1974) 535; Annals Phys. 91 (1975) 75. 
[8] J. D. Bekenstein, Phys. Rev. D 51 (1995) no.12, R6608.

[9] C. A. R. Herdeiro and E. Radu, Int. J. Mod. Phys. D 24 (2015) no.09, 1542014.

[10] T. P. Sotiriou and V. Faraoni, Phys. Rev. Lett. 108 (2012) 081103.

[11] L. Hui and A. Nicolis, Phys. Rev. Lett. 110 (2013) 241104.

[12] P. Kanti, N. E. Mavromatos, J. Rizos, K. Tamvakis and E. Winstanley, Phys. Rev. D 54 (1996) 5049; Phys. Rev. D 57 (1998) 6255.

[13] T. Torii, H. Yajima and K. i. Maeda, Phys. Rev. D 55 (1997) 739.

[14] P. Kanti and K. Tamvakis, Phys. Lett. B 392 (1997) 30.

[15] B. Kleihaus, J. Kunz and E. Radu, Phys. Rev. Lett. 106 (2011) 151104.

[16] P. Pani, C. F. B. Macedo, L. C. B. Crispino and V. Cardoso, Phys. Rev. D 84 (2011) 087501.

[17] E. Babichev and C. Charmousis, JHEP 1408 (2014) 106.

[18] T. P. Sotiriou and S. Y. Zhou, Phys. Rev. Lett. 112 (2014) 251102; Phys. Rev. D 90 (2014) 124063.

[19] G. Antoniou, A. Bakopoulos and P. Kanti, Phys. Rev. Lett. 120 (2018) no.13, 131102; Phys. Rev. D 97 (2018) no.8, 084037.

[20] A. Bakopoulos, G. Antoniou and P. Kanti, Phys. Rev. D 99 (2019) no.6, 064003 doi:10.1103/PhysRevD.99.064003 [arXiv:1812.06941 [hep-th]].

[21] A. Bakopoulos, G. Antoniou and Panagiota Kanti, AIP Conf. Proc. 2075 (2019) no.1, 040003.

[22] T. j. Chen, M. Fasiello, E. A. Lim and A. J. Tolley, JCAP 1302 (2013) 042.

[23] I. Antoniadis, J. Rizos and K. Tamvakis, Nucl. Phys. B 415 (1994) 497.

[24] P. Kanti, B. Kleihaus and J. Kunz, Phys. Rev. Lett. 107 (2011) 271101; Phys. Rev. D 85 (2012) 044007.

[25] J. L. Blazquez-Salcedo et al., IAU Symp. 324 (2016) 265 [arXiv:1610.09214 [gr-qc]].

[26] Y. Brihaye, B. Hartmann and J. Urrestilla, JHEP 1806 (2018) 074; Y. Brihaye and B. Hartmann, arXiv: 1810.05108 [gr-qc].

[27] J. Rizos and K. Tamvakis, Phys. Lett. B 326 (1994) 57.

[28] P. Kanti, J. Rizos and K. Tamvakis, Phys. Rev. D 59 (1999) 083512.

[29] P. Kanti, R. Gannouji and N. Dadhich, Phys. Rev. D 92 (2015) no.4, 041302; Phys. Rev. D 92 (2015) no.8, 083524.

[30] D. D. Doneva and S. S. Yazadjiev, Phys. Rev. Lett. 120 (2018) no.13, 131103.

[31] H. O. Silva et al., Phys. Rev. Lett. 120 (2018) no.13, 131104.

[32] S. Bhattacharya and S. Chakraborty, Phys. Rev. D 95 (2017) no.4, 044037; I. Banerjee,

S. Chakraborty and S. SenGupta, Phys. Rev. D 96 (2017) no.8, 084035.

[33] J. L. Blazquez-Salcedo, C. F. B. Macedo, V. Cardoso, V. Ferrari, L. Gualtieri, F. S. Khoo, J. Kunz and P. Pani, Phys. Rev. D 94 (2016) no.10, 104024.

[34] A. Bakopoulos, P. Kanti and N. Pappas, in preparation. 